\title{
Le règlement européen " essais cliniques »: articulation avec la loi Jardé : un atelier de Giens
}

François Lemaire ${ }^{1}$, Brigitte Marchenay ${ }^{2}$, Olivier Chassany ${ }^{3}$ et les participants à la table ronde $N^{\circ} 2$ de Giens XXX : Philippe Barthélémy ${ }^{4}$ Mohamed Bouzzagou ${ }^{5}$, Denis Comet ${ }^{6}$, Cécile Delval ${ }^{7}$, Claude Dubray ${ }^{8}$, Cécile Fouret ${ }^{9}$, Elisabeth Frija-Orvoen ${ }^{10}$, Laetitia Gambotti ${ }^{11}$, Véronique Lamarque ${ }^{12}$, Geneviève d'Orsay ${ }^{13}$, Valérie Plattner ${ }^{14}$, Claire Sibenaler ${ }^{15}$, Jacques Roux ${ }^{16}$ et Frédérique Thoby ${ }^{17 \dagger}$

1 Professeur Emérite Université Paris Est, Créteil, France

2 Laboratoire Roche, Boulogne Billancourt, France

3 Université Paris Diderot, Assistance Publique - Hôpitaux de Paris, Paris, France

4 Laboratoire AstraZeneca, Rueil Malmaison, France

5 Laboratoire Boehringer-Ingelheim, Reims, France

6 AFCROs, Axonal, Nanterre, France

7 ANSM, Saint Denis la Plaine, France

8 Centre d'Investigation Clinique, Clermont-Ferrand, France

9 Medtronic, Boulogne Billancourt, France

10 CNCPP, Paris, France

11 Assistance Publique - Hôpitaux de Paris, Paris, France

12 Eval Santé, Croissy-sur-Seine, France

13 Voluntis, Suresnes, France

14 Hospices Civils, Lyon, France

15 LEEM, Les Entreprises du Médicament, Paris, France

16 Laboratoire Glaxosmithkline, Marly-le-Roi, France

17 Laboratoire Novartis, Rueil Malmaison, France

Texte reçu le 14 novembre 2014 ; accepté le 6 décembre 2014

\begin{abstract}
Mots clés :
règlement européen ;

essais cliniques

Résumé - Le Parlement et le Conseil de l'Union Européenne ont publié en mai 2014 un nouveau règlement relatif aux essais de médicaments ; cliniques de médicaments, destiné à remplacer la directive 2001/20/CE. Sa mise en application n'interviendra pas avant 2016. Néanmoins, il est essentiel de se préoccuper dès à présent de l'articulation de ce texte avec la législation nationale, c'est-àdire de la loi Jardé dont la mise en œuvre a été retardée, précisément dans l'attente de la publication du règlement européen. transparence ; évaluation L'atelier de Giens a listé et étudié les différents problèmes que cette articulation ne manquera pas de poser. Ont été partiméthodologique des culièrement abordés : les modalités d'évaluation de la méthodologie des essais, l'articulation fonctionnelle entre l'Agence demandes d'autorisation; Nationale de Sécurité du Médicament et des Produits de Santé (ANSM) et les Comités de Protection des Personnes (CPP) loi Jardé ; loi pendant cette phase d'évaluation des demandes d'autorisation, l'évaluation des études post-autorisation/inscription des méinformatique et libertés ; dicaments et des dispositions médicaux, la transparence des données.
\end{abstract}

CPP ; ANSM ;

dispositifs médicaux

Abréviations : voir en fin d'article.

\footnotetext{
$†$ Les articles, analyses et propositions des Ateliers de Giens n’engagent que leurs auteurs et ne préjugent pas de la position de leur organisme de tutelle.
} 
Tableau I. Règlement européen : dispositions essentielles.

- À la différence d'une directive, le règlement s'impose à tous les EM de l'Union Européenne sans possibilité de le modifier

- Champ : études interventionnelles sur le médicament

- Demande d'autorisation : choix de l'EM rapporteur proposé par le promoteur

- Deux évaluations distinctes : 1- scientifique (partie I) : évaluation coordonnée entre EM concernés ; 2 - éthique (partie II) : évaluation par chaque EM

- Un avis unique donné par un EM rapporteur unique pour la partie I et une décision nationale d'autorisation unique par EM (conclusion de l'évaluation de la partie I + II), prise par l'autorité compétente

- Portail et base de données uniques (EMA)

- Autorisation tacite après 60 jours pour une demande initiale (91 jours si questions)

- Une nouvelle catégorie de recherche «avec faible niveau d'intervention » (approche basée sur le risque - risk-based approach); avantages : traçabilité, vigilance et monitoring allégés.

- Consentement : dérogation possible si urgence ou clusters, accord global (consentement pour utilisation ultérieure des données), données possiblement gardées en cas de retrait du consentement, sauf opposition

- Suppression de l'envoi des EIG aux CPP

- Délai fixe de réponse des promoteurs aux questions émises par le CPP

- Possibilité de co-promotion

- Transparence et accès public des données cliniques

CPP : Comité de Protection des Personnes; EIG : effets indésirables graves ; EM : Etats Membres ; EMA : Agence Européenne du Médicament.

\section{Introduction}

Le 27 mai 2014, le journal officiel de l'Union Européenne (UE) publiait un nouveau règlement sur les essais cliniques portant sur les médicaments. ${ }^{[1]} \mathrm{Ce}$ règlement était destiné à remplacer la directive 2001/20/CE, elle-même transposée dans tous les Etats Membres (EM) de l'Union. Pour la France, cette transposition a été réalisée en 2004, dans la loi de santé publique qui révisait la loi Huriet-Sérusclat. Près de dix ans après la mise en application de la directive 2001/20, la Commission Européenne a entrepris d'évaluer son impact sur la conduite de la recherche clinique en Europe. ${ }^{[2]}$ Rejoignant le constat déjà réalisé par l'industrie pharmaceutique et les promoteurs académiques, ${ }^{[3]}$ le bilan était inquiétant. La Commission devait constater en effet qu'entre 2007 et 2011, le nombre d'essais cliniques portant sur des médicaments en Europe avait chuté de $25 \%$, alors que les coûts avaient doublé et que les délais de démarrage avaient augmenté de $90 \%$ ! Décidée à remédier à cet état de choses, la Commission a publié en juillet 2012 un projet de règlement, porteur d'amélioration et de simplification de l'encadrement qu'elle avait elle-même contribué à élaborer. Ensuite, pendant toute la durée de l'année 2013, ce projet a été étudié et amendé par le Conseil des EM et le Parlement Européen. Un compromis entre les trois textes a finalement été trouvé à Strasbourg en décembre 2013, suffisamment à temps pour que le règlement soit voté lors de la dernière session du Parlement, en avril 2014, juste avant les nouvelles élections.

Le nouveau règlement sera applicable dans le second semestre 2016. D'ici là, il devra avoir été intégré dans les différentes législations nationales de l'UE. En France, cela implique que le règlement ait été harmonisé avec la loi Jardé. Or, celle-ci, quoique publiée en mars 2012, n'est toujours pas applicable, dans l'attente de son décret d'application. Une table ronde des Ateliers de Giens a été consacrée à la mise en œuvre du règlement européen « essais cliniques » et à sa nécessaire articulation avec la loi Jardé.

\section{Principales dispositions du règlement européen et de la loi Jardé}

Les principales dispositions du règlement européen et de la loi Jardé sont résumées dans les tableaux I et II.

\section{Articulation du règlement européen et de la loi Jardé : mise en œuvre et calendrier}

L'intégration du règlement européen dans le droit national nécessite une intervention législative. Pour ce faire, le gouvernement a décidé de légiférer par ordonnance, ce qui supprimera le débat parlementaire. Néanmoins, les députés doivent en accepter le principe. Le projet de loi de santé de Marisol Touraine comprend un article d' « habilitation» qui autorisera le gouvernement à «...adapter la législation relative aux recherches biomédicales définies au titre II du livre $I^{\text {er }}$ de la première partie du code de la santé publique au règlement (UE) $n^{\circ} 536 / 2014 \ldots$, d'adapter cette législation aux fins de coordonner l'intervention des Comités de Protection des Personnes mentionnés à l'article L. 1123-1 du code de la Santé Publique et de procéder aux modifications de la même législation lorsque des adaptations avec d'autres dispositions législatives sont nécessaires. » 
Tableau II. Dispositions essentielles de la loi Jardé.

- Le champ de la loi couvre la totalité des recherches impliquant la personne humaine, y compris les recherches non interventionnelles ; la loi française encadre aussi les recherches hors médicaments (depuis la loi Huriet de 1988)

- Application de l'approche basée sur le risque : trois catégories de recherche : 1. interventionnelle 2. à risque minime 3 . non interventionnelle (RNI)

- Toutes les recherches impliquant la personne humaine sont soumises au CPP; celui-ci étudie aussi les aspects liés à la protection des données personnelles, en amont de la soumission à la CNIL

- Consentement : exprès (écrit ou oral, en fonction du contexte et de l'accord du $\mathrm{CPP}$ ) pour les recherches à risque minime, dérogation si «risque vital immédiat», information et non opposition si RNI, dérogation possible si clusters.

- CPP : création d'une commission nationale d'harmonisation, placée au Ministère de la Santé, sans rôle opérationnel ; tirage au sort des comités

- Clarification concernant les recherches portant sur les collections d'échantillons biologiques et les recherches génétiques

CNIL : Commission Nationale Informatique et Libertés; CPP : Comité de Protection des Personnes ; RNI : recherche non interventionnelle.

Tableau III. Evaluation de la partie I (article 6 du règlement).

a. Validation de la qualification de l'essai : le cas échéant, essai à faible niveau d'intervention

b. Conformité au chapitre $\mathrm{V}$ du règlement (protection des participants et consentement éclairé) :

1. Evaluation des bénéfices sur le plan thérapeutique et de la santé publique :

- caractéristiques des médicaments expérimentaux et auxiliaires;

- pertinence de l'essai clinique : représentativité des groupes de participants, état des connaissances scientifiques;

- fiabilité, robustesse des données : méthodologie (taille échantillon, randomisation, comparateur, critères d'évaluation).

2. Risques et inconvénients pour les participants :

- caractéristiques des médicaments expérimentaux et des auxiliaires ;

- caractéristiques de l'intervention par rapport à la pratique ;

- risques pour la santé des participants.

c. Fabrication des médicaments expérimentaux et auxiliaires

d. Etiquetage

e. Brochure investigateur

Il existera en France deux législations différentes, l'une relative aux essais interventionnels portant sur les médicaments, le règlement, et l'autre relative aux autres recherches, la loi Jardé.

L'objet de la table ronde $\mathrm{N}^{\circ} 2$ des Atelier de Giens était d'envisager les problèmes que pourrait poser cette coexistence, certains domaines devant nécessairement rester communs.

\section{Evaluation de la méthodologie des essais cliniques : CPP ou ANSM?}

Le règlement européen a découpé l'évaluation du dossier de demande d'autorisation en deux parties, la première consacrée à l'évaluation scientifique et technique (partie I), la deuxième à l'évaluation éthique (partie II), comme il est résumé dans les tableaux III et IV. Même si cela n'est pas écrit explicitement dans le règlement (la Commission a expliqué à de nombreuses reprises que l'éthique ne figure pas dans le champ communautaire et reste de compétence nationale, elle s'interdit donc de légiférer dans ce domaine), l'interprétation la plus commune de cette législation est que l'évaluation de la partie I sera effectuée par les autorités compétentes, en France l'Agence Nationale de Sécurité du
Médicament et des Produits de Santé (ANSM), et celle de la partie II par les «comités d'éthique de la recherche», en France les Comités de Protection des Personnes (CPP).

Le règlement a ainsi prévu de confier l'évaluation scientifique, des projets de recherche à l'autorité compétente, alors qu'elle est aujourd'hui réalisée en France par les CPP. Cependant, il est aussi clairement indiqué à l'article 4 que les comités d'éthique peuvent prendre en compte dans leur évaluation certains éléments de la partie I. Le considérant 18 ne laisse aucune ambiguité sur ce point ${ }^{1}$.

Les arguments du débat en France sont les suivants :

1. Pour les représentants des CPP, l'argument essentiel est que les analyses éthique et scientifique d'un protocole sont étroitement mêlées et que l'on ne peut réaliser une expertise éthique d'un essai indépendamment de son contexte scientifique. Une

1 Considérant 18 : «Il convient de laisser aux États membres le soin de désigner l'organe ou les organes appropriés participant à l'évaluation de la demande de participation à un essai clinique ainsi que d'organiser la participation de comités d'éthique dans les limites du calendrier fixé par le présent règlement pour l'autorisation de l'essai clinique. De telles décisions relèvent de l'organisation interne de chaque État membre... » 
Tableau IV. Evaluation de la partie II (article 7 du règlement).
a. Consentement
b. Modalités de rétribution/indemnisation des participants et des investigateurs
c. Modalités de recrutement des participants
d. Protection données personnelles
e. Aptitude des investigateurs
f. Adéquation des sites d'investigations
g. Compensation des dommages liés à l'essai
h. Règles applicables aux collections biologiques

importante littérature scientifique abonde dans ce sens ${ }^{2}$. La réglementation en vigueur en France le reconnaît d'ailleurs implicitement puisqu'elle impose la présence d'un épidémiologiste ou d'un biostatisticien dans le collège 1 des comités. L'ANSM a toujours admis cette division des tâches telles que définies dans la loi de Santé Publique de 2004 et limité son expertise à la sécurité des produits expérimentaux. Les CPP s'acquittent de cette mission d'analyse méthodologique des protocoles depuis plus de vingt ans, et ils ont acquis à cet égard une expertise reconnue. De plus, cette évaluation scientifique constitue une motivation pour les membres du collège 1 , un facteur majeur d'attractivité pour les CPP, et leur retirer cette mission risque de supprimer cette incitation. Mais on reproche aux CPP leur lenteur et leur manque de réactivité ; dans un contexte où l'avis unique délivré par l'Etat Membre (l'autorité compétente, l'ANSM en France) comprend l'évaluation des parties I et II, l'ANSM craint que les comités soient incapables de fournir leur avis dans les délais imposés par le règlement : 26 jours quand la France sera EM rapporteur, 45 jours dans les autres cas. La concertation entre les différents EM concernés par l'essai pourrait être aussi difficile si une partie du rapport d'évaluation n'a pas été effectuée par l'ANSM. A cela, les représentants de la Conférence des Présidents de Comités de Protection des Personnes (CNCP) répondent que les CPP sont conscients qu'ils vont devoir adapter leurs méthodes de travail et qu'ils pourront tenir les délais exigés.

2. L'ANSM met en avant la nécessité d'éviter à tout prix les doublons dans les évaluations de l'Agence et des CPP, et le respect des délais. En raison du maintien dans le règlement des dispositions dites de l' «accord tacite », tout essai dont l'évaluation n'aura pas été terminée à 60 jours serait automatiquement autorisé (article 8-4). L'ANSM doute que, compte tenu de l'extrême diversité des CPP sur le territoire et de l'hétérogénéité de leurs modes d'évaluation, ceux-ci soient en mesure de respecter

\footnotetext{
${ }^{2}$ A noter que ce débat en France a été largement occulté par l'interdit énoncé dès la première rédaction de la loi Huriet : les Comités de Protection des Personnes ne seraient en France «ni scientifiques, ni éthiques », comme l'avait déclaré à l'Assemblée Nationale Jean-François Mattei, Ministre de la Santé à l'époque ! ${ }^{[4,5]}$
}

les délais très courts imposés par le règlement, au moins lorsque la France sera rapporteur. Ce constat est partagé par les promoteurs industriels. Ensuite, l'Agence entend l'argument selon lequel elle ne saurait en l'état actuel de ses capacités d'expertise (peu ou pas de méthodologistes en son sein) évaluer le contenu scientifique des protocoles de recherche. Cependant, elle fait remarquer qu'elle développe déjà cette expertise lors de l'évaluation des demandes d'autorisation de mise sur le marché (AMM), d'autorisation temporaire d'utilisation (ATU)... et que pour l'instruction des demandes d'autorisation des essais cliniques, elle envisage de s'appuyer sur le groupe de travail externe (GTE) recherche biomédicale, déjà nommé par le Directeur Général de l'Agence, et qui pourra le cas échéant être complété par des experts méthodologistes. L'ANSM précise que c'est elle qui délivrera l'autorisation de la demande pour l'Etat membre qu'elle en a la responsabilité, et qu'à ce titre elle entend en garder la maîtrise totale.

Discussion. À ce jour (novembre 2014), l'arbitrage final sur ce point crucial n'est pas connu. La table ronde de Giens admet cependant que même si l'évaluation méthodologique devait échoir finalement à l'ANSM (le plus probable), les CPP ne s'interdiront pas de « regarder» la méthodologie des essais, ce « regard» étant nécessaire pour une bonne évaluation de la qualité de l'information des participants. L'essentiel sera surtout d'établir un dialogue efficace et réactif entre les CPP et l'Agence pendant toute la phase d'évaluation, afin d'éviter un blocage final. Les modalités de cette concertation devront être mises au point pendant la phase pilote organisée par l'Agence.

\section{Le choix de la langue dans laquelle seront rédigés les dossiers de demande d'autorisation}

La procédure d'autorisation se déroulera intégralement sur le portail européen, sur lequel seront «postés » la totalité du dossier (protocole, brochure investigateur), les éléments de suivi (modifications substantielles, etc) et les résultats (résumé, rapport d'étude clinique). C'est aussi par ce portail que s'effectueront les échanges au sein des EM concernés. L'article 26 du règlement se contente d'énoncer : "La langue du dossier de demande, ou de parties de celui-ci, est déterminée par l'État Membre concerné. Pour l'application du premier alinéa, les États Membres envisagent la possibilité d'accepter, pour les documents non destinés au participant, une langue communément comprise dans le secteur médical. » Mais la «langue communément comprise dans le secteur médical » ne peut bien évidemment qu'être l'anglais. Deux logiques s'affrontent donc : la volonté de mettre à disposition un outil permettant la compréhension et les échanges (entre les autorités compétentes et les promoteurs) à l'échelle européenne, multinationale, qui impose l'anglais, d'une part; et, d'autre part, les 
nécessités concernant les échanges entre les autorités compétentes nationales, les comités d'éthiques et les promoteurs/investigateurs (voire les participants aux essais, en ce qui concerne les documents d'information et de consentement) dont une partie au moins ne peut se faire qu'en langue nationale. Par exemple, une partie importante de l'avis des CPP concerne la rédaction des documents d'information et de consentement. On voit mal comment ces recommandations pourraient se faire en France comme au niveau de tous les Etats Membres non anglophones dans une langue autre que la langue nationale?

Discussion. Il semble raisonnable que les rapports d'évaluation, aussi bien de la partie I que de la partie II, soient rédigés en anglais, de même que la notification de l'autorisation de l'ANSM au promoteur. Le résumé/conclusion du CPP au promoteur (les questions) pourrait être rédigé en anglais, mais néanmoins les questions et commentaires se rapportant spécifiquement à la rédaction des notices d'informations et des formulaires de consentement devraient être rédigés en français. Le promoteur répondrait globalement en anglais, sauf sur les parties rédigées en français.

\section{L'évaluation éthique (partie II) par les CPP}

La loi Jardé introduit plusieurs modifications importantes concernant les missions des CPP : l'avis sur les demandes d'autorisation des recherches non interventionnelles « impliquant la personne humaine », la protection des données personnelles (avant soumission à la Commission Nationale Informatique et Libertés [CNIL]), la dérogation au consentement dans plusieurs situations particulières (recherche en situation d' « urgence vitale immédiate ", recherches génétiques portant sur des échantillons provenant de personnes décédées,...). Cet élargissement du champ d'intervention des CPP va mécaniquement entrâner une augmentation des dossiers traités. A laquelle va s'ajouter la nouvelle contrainte des délais serrés qu'imposera le règlement européen à partir de 2016 (45 jours pour l'évaluation de la partie II par les CPP). Enfin, la loi Jardé va aussi modifier le mode d'attribution des dossiers aux CPP, qui se fera par tirage au sort. Or, la plupart des comités ne se réunissent pas au mois d'août et la quasi-totalité d'entre eux n'ont qu'une réunion mensuelle. Il est à noter que dans le choix que les firmes pharmaceutiques font du CPP où déposer une demande d'avis, la proximité de la date de la prochaine réunion est la variable prédominante!

À l'évidence, l'organisation du fonctionnement des comités va devoir être repensée. De nombreuses pistes ont été proposées et discutées :

\section{Discussion.}

1. Augmenter la fréquence des réunions, à deux par mois, comme quelques rares Comités le font déjà; cette recommandation a très peu de chances d'être suivie; il faut rappeler ici que les membres des Comités sont des bénévoles et qu'il sera difficile d'augmenter leur temps de présence en réunion (hormis le cas des retraités...).

2. Organiser dans chaque Comité une voie «accélérée » d'analyse des dossiers les plus simples (par exemple, la plupart des recherches observationnelles). L'expedited review est déjà mise en œuvre par les comités nord-américains (institutional review board $[\mathrm{IRB}]$ ), autrichiens, anglais. ${ }^{[6]}$ Le principe général est que les dossiers réputés sans problème au départ sont confiés par le président du Comité à un ou deux rapporteurs, qui lui retournent leur évaluation. Si leur avis est favorable, le bureau prend une décision immédiate, et le président en informe le Comité à l'occasion de la séance plénière suivante. Dans le cas contraire (erreur d'aiguillage ou recherche s'avérant problématique), le dossier est reversé dans le pool des demandes traitées par la voie habituelle. Cette proposition figurait déjà dans le projet de décret modificatif mis en circulation par la Direction Générale de la Santé (DGS) en novembre 2012.

3. Organiser un calendrier des Comités sur une base interrégionale. Probablement une des suggestions les plus intéressantes; au sein de chaque inter région, la réunion d'un Comité serait assurée chaque semaine, selon un ordre prédéterminé. Ainsi, chaque dossier de soumission pourrait être traité par un Comité en quelques jours seulement. Inconvénient : l'ordre de réunion étant connu à l'avance, cette organisation est incompatible avec la « distribution aléatoire » de la loi Jardé. .

4. Les Comités ont 45 jours pour évaluer la partie II dans le cadre de la demande initiale (avec ou sans la méthodologie, voir plus haut); il faut par conséquent que le système de communication entre l'ANSM et les CPP concernés fonctionne rapidement, dès qu'une demande d'autorisation aura été déposée, afin que les Comités puissent valider la partie II et débuter l'expertise du dossier le plus vite possible ; c'est un des points que devrait traiter en priorité la phase pilote organisée par l'ANSM.

5. Mise en activité rapide de la Commission nationale destinée à harmoniser le fonctionnement des CPP prévue par la loi Jardé.

\section{Etudes post-autorisation observationnelles demandées par les autorités de santé}

Depuis la loi Bertrand en France ${ }^{[7]}$ et son décret d'application $^{[8]}$ ou la nouvelle législation pharmaceutique ${ }^{[9,10]}$ au niveau européen, les autorités de santé peuvent imposer aux titulaires d'autorisation de mise sur le marché la réalisation d'études observationnelles ou non, d'efficacité ou de sécurité post-autorisation, notamment dans le cadre des plans de gestion des risques. 
Une étude de sécurité post-autorisation (post-authorization safety study [PASS] peut être requise s'il existe des craintes quant aux risques de sécurité posés par un médicament autorisé, avec pour objectif d'identifier, décrire ou quantifier un risque, de confirmer le profil de sécurité du médicament ou de mesurer l'efficacité des mesures de gestion des risques.

Quant aux études d'efficacité post-autorisation (postauthorization efficacy study [PAES]), elles peuvent être requises lorsque la compréhension de la maladie ou la méthodologie clinique suggère que les évaluations d'efficacité antérieures méritent d'être revues significativement.

Par ailleurs depuis plusieurs années en France, lors de l'évaluation des médicaments avant la commercialisation, la Commission de la Transparence peut demander au laboratoire de conduire une étude post-inscription afin de connaître, en situation réelle, les modalités d'utilisation, le bénéfice et l'impact du produit dans la vraie vie. C'est également le cas pour le dispositif médical (DM) pour lequel des études post-inscription peuvent être demandées par la Commission Nationale d'Evaluation des Dispositifs Médicaux et des Technologies de Santé (CNEDIMTS) ou le Comité Economique des Produits de Santé (CEPS). Toutes ces études bénéficient d'une validation formelle par l'autorité, qu'il s'agisse de l'Agence Européenne du Médicament (EMA), de l'ANSM ou de la Haute Autorité de Santé (HAS).

Or dans la loi Jardé (loi n 2012-300 du 5 mars 2012) relative aux recherches impliquant la personne humaine, il est précisé que les recherches non interventionnelles ne peuvent être mises en œuvre qu'après avis favorable du CPP. La seule exonération introduite concerne l'information des participants ${ }^{3}$.

Discussion. Afin de rendre le système d'évaluation efficace et non redondant, il serait approprié de préciser que toute recherche non interventionnelle répondant à une obligation de l'Agence Nationale de Sécurité du Médicament et des Produits de Santé, de la Haute Autorité de Santé ou de l'Agence Européenne des Médicaments, ne sera soumise au CPP que pour l'évaluation éthique, dès lors qu'une approbation méthodologique aura été octroyée par les autorités concernées.

\section{Révision de la loi informatique et libertés (니)}

La CNIL annonçait depuis de nombreuses années sa volonté de réviser la LIL. Cette révision, préparée en 2013 par un rapport

\footnotetext{
3 Article L1122-1 (CSP) : "Lorsqu'une recherche non interventionnelle porte sur l'observance d'un traitement et que sa réalisation répond à une demande de l'Agence nationale de sécurité du médicament et des produits de santé, de la Haute Autorité de santé ou de l'Agence européenne des médicaments, l'objectif de la recherche, sa méthodologie et sa durée peuvent ne faire l'objet que d'une information préalable succincte dès lors que la recherche ne présente aucun risque sérieux prévisible. »
}

de l'Inspection Générale des Affaires Sociales (IGAS) ${ }^{4}{ }^{[11]}$ est actuellement portée par la loi de santé de Marisol Touraine. Celle-ci comprend trois volets destinés à :

1. Unifier les dispositions qui régissent les traitements de données issues du soin (chapitre $\mathrm{X}$ de la LIL) ou de la recherche (chapitre IX).

2. Faciliter l'accès aux chercheurs des données médicoadministratives, type Système National d'Information Interrégimes de l'Assurance Maladie (SNIIRAM) ou Programme de Médicalisation des Systèmes d'Information (PMSI).

3. Clarifier les relations entre la loi Jardé et la LIL en ce qui concerne le périmètre couvert par chacun des deux textes et les structures d'autorisation et de contrôle (CPP et CCTIRS). C'est ce dernier point qui nous intéresse ici. Le champ d'application de la loi Jardé concerne les recherches «impliquant la personne humaine », y compris donc toutes les recherches non interventionnelles (article L1121-1-3); ces recherches doivent être soumises à un $\mathrm{CPP}$, dont les missions ont été modifiées en conséquence par la loi Jardé. En revanche, les recherches sur données existantes, et qui correspondent donc à un changement de finalité, restent dans le champ de la LIL et doivent être soumises au CCTIRS.

En matière de protection des données, l'avis, que ce soit celui du CPP ou celui du CCTIRS, est un avis destiné à éclairer la CNIL, qui doit être saisie en aval. Pour les recherches «impliquant la personne humaine », c'est dire toute l'importance des méthodologies de référence qui s'appliquent aux traitements de données les plus courants et qui permettent d'éviter le passage par la CNIL : la MR 001, aujourd'hui, peut s'appliquer aux recherches «biomédicales ».

Discussion. Les membres de la table ronde ont pris acte de cette clarification. A notamment été évoquée, la possibilité pour la CNIL, dans le plan de simplification administrative mise en place par le gouvernement, de passer du refus à l'autorisation tacite en cas de dépassement des délais (comme cela est prévu par le règlement pour l'autorisation d'un essai clinique).

Il a par ailleurs été proposé que la CNIL étende le champ de ces méthodologies de référence, notamment aux recherches avec risque minime, certaines recherches non interventionnelles, les études post-autorisation/post inscription concernant les médicaments (PASS/PAES) et les dispositifs médicaux, etc.

\footnotetext{
${ }^{4}$ Le rapport de l'IGAS sur les traitements de données (P.-L. Bras et A. Loth), ${ }^{[11]}$ relayé ensuite par un autre rapport IGAS de 2014, consacré aux $\mathrm{CPP}^{[12]}$ avait proposé que la totalité des recherches non interventionnelles soient soumises au Comité Consultatif sur le Traitement de l'Information en matière de Recherche (CCTIRS) et non pas aux CPP. Ce n'est pas la solution qui a été finalement retenue.
} 


\section{Transparence}

Une des avancées importantes du règlement est l'obligation de rendre la base de données européenne accessible librement aux chercheurs et plus largement au public, sauf pour tout ou partie des données et informations pour lesquelles il convient de conserver une confidentialité. Les considérants 67 et 68 et l'article 81 du règlement précisent que cette transparence vise à permettre la coopération entre les autorités compétentes des EM, et entre promoteurs et EM concernés (autorité compétente + comité d'éthique). Mais elle doit aussi participer à l'information du public. «Toutes » les données consignées dans la base de données seront accessibles, ce qui devrait correspondre au protocole, à la brochure investigateur, aux demandes de modification substantielle, au rapport d'essai clinique et aux résumés. Aucune information à caractère personnel ne sera transmise, de même que les informations confidentielles à caractère commercial (données de fabrication, par exemple). Les articles 36 et 37 du règlement précisent les délais dans lesquels le début et la fin de l'essai doivent être notifiés dans la base de données de l'EMA par le promoteur (dans les 15 jours). Quels que soient les résultats de l'essai clinique, un résumé des résultats doit être transmis dans la base de données et ce dernier doit être accompagné d'un résumé en langage facilement compréhensible. De plus, lorsque l'essai est associé à une demande d'autorisation de mise sur le marché d'un médicament expérimental, le rapport d'étude clinique doit être transmis dans la base dans un délai de trente jours consécutifs à la décision.

En parallèle, et afin de ne pas attendre la mise en ouvre du règlement qui n'aura pas lieu avant 2016, l'EMA a adopté une politique concernant la publication des données sur les essais cliniques qui s'appliquera à partir du $1^{\mathrm{er}}$ janvier 2015 pour les nouvelles demandes et du 15 juillet pour les amendements.

Discussion. Les dispositions pratiques concernant la transparence de la base de données du règlement méritent d'être précisées, notamment leur articulation avec la politique de transparence qui vient d'être adoptée par l'EMA le 2 octobre dernier et qui précisent les conditions dans lesquelles les rapports des essais cliniques des dossiers de demande d'AMM seront accessibles au public, aux chercheurs, etc.

\section{Reprendre dans la loi française certaines avancées du règlement européen}

1. Le consentement global : le participant peut consentir d'emblée, dès l'inclusion dans l'essai, à ce que les résultats de celui-ci soient utilisés dans le futur pour d'autres recherches, sans qu'il soit nécessaire de l'informer à nouveau (Article 28-2).
2. Si le participant retire son consentement, l'utilisation des données obtenues avant ce retrait reste possible, sans préjudice des dispositions concernant la protection des données (Article 28-3)

3. De même, si un participant a été inclus en urgence, sans consentement préalable, et que redevenu capable de consentir, il s'oppose à la poursuite de l'essai, l'utilisation des données obtenues avant ce refus est possible, sauf si le participant s'y oppose (Article 35-3)

4. L'allégement de la vigilance (non déclaration de l'investigateur au promoteur de certains évènements indésirables graves [EIG]) dans les recherches avec faible intervention (Article 41-2)

5. Suppression de l'obligation de notification des EIG au CPP par le promoteur.

\section{Phase pilote}

Afin d'anticiper la mise en application du règlement européen, l'ANSM met en place une phase pilote dont les objectifs sont l'optimisation des délais, l'articulation, le partage des responsabilités avec le comité d'éthique. Pour ce faire, elle a constitué un groupe de travail avec des représentants des CPP, de la DGS, des promoteurs académiques et industriels.

Discussion. Lors des réunions de préparation de la phase pilote organisée par l'ANSM, les points suivants méritent d'être particulièrement étudiés :

1. L'organisation du dialogue entre le CPP et l'Agence.

2. Des modèles-type de documents et d'avis devraient être rédigés.

3. Un de ces documents-type devrait concerner les dispositions financières de l'essai à destination du CPP; il devrait rester très général.

4. L'avis aux promoteurs d'essais cliniques sera prochainement mis à jour et un avis spécifique à la phase pilote créé.

5. Mise au point des modalités d'appel en cas d'avis négatif du CPP.

\section{Dispositifs médicaux}

Au plan européen, deux textes encadrent la mise en œuvre des essais cliniques : le règlement européen concernant les essais de médicaments d'ores et déjà publié et celui à venir sur les DM. La loi Jardé a vocation, elle, à couvrir l'ensemble des recherches menées sur l'être humain, quels que soient le type de produit ou la technique testée. En ce sens, la loi Jardé doit permettre la réalisation dans des conditions claires de tous les essais, quelle que soit la catégorie de produits concernée. 
Le règlement européen concernant les dispositifs médicaux, quant à lui, est actuellement en cours de discussion dans le cadre du trialogue entre la Commission Européenne, le Parlement et le Conseil. Sa publication est attendue courant septembre 2015 pour une mise en application 3 ans plus tard. Contrairement au médicament, ce règlement ne couvre pas uniquement la réalisation des essais cliniques, mais toute la réglementation concernant les dispositifs médicaux (organismes notifiés, marquage CE, classification, matériovigilance, suivi post-marché, essais...). Pour autant, les dispositions propres à la mise en place des essais cliniques DM seront intégrées dans ce texte, et le principe sera le même que pour le médicament (une autorisation gérée par un EM de référence). L'ANSM suit ce point actuellement pour veiller à la cohérence des deux règlements en termes d'essai clinique.

Discussion. Le groupe recommande donc de suivre attentivement l'évolution du texte DM afin de permettre une bonne articulation avec le texte médicament. Il recommande également, pour les éléments qui seront spécifiquement nationaux, de prendre en compte dans les textes, les spécificités du DM pouvant influer sur la gestion des essais cliniques.

Conflits d'intérêts. Aucun.

Abréviations. AMM : autorisation de mise sur le marché; ANSM : Agence Nationale de Sécurité du Médicament et des Produits de Santé ; ATU : Autorisation Temporaire d'Utilisation; CCTIRS : Comité Consultatif sur le Traitement de l'Information en matière de Recherche; CEPS : Comité Economique des Produits de Santé; CNCP : Conférence des Présidents de Comités de Protection des Personnes; CNEDIMTS : Commission Nationale d'Evaluation des Dispositifs Médicaux et des Technologies de Santé ; CNIL : Commission Nationale Informatique et Libertés ; CPP : Comité de Protection des Personnes; DGS : Direction Générale de la Santé ; DM : Dispositif Médical ; EIG : effets indésirables graves ; EM : Etats Membres ; EMA : Agence Européenne du Médicament; GTE : groupe de travail externe; HAS : Haute Autorité de Santé ; IGAS : Inspection Générale des Affaires Sociales; IRB : institutional review board; LIL : loi informatique et libertés; PASS : post-authorization safety study; PAES : postauthorization efficacy study; PMSI : Programme de Médicalisation des Systèmes d'Information; SNIIRAM : Système National d'Information Inter Regimes de l'Assurance Maladie ; UE : Union Européenne.

\section{Références}

1. European Parliament and Council. Regulation (EU) No 536/2014 of the European Parliament and of the Council of 16 April 2014 on clinical trials on medicinal products for human use, and repealing Directive 2001/20/EC. 2014 http://eur-lex. europa.eu/legal-content/EN/TXT/? uri=uriserv:0].L_.2014.158.01.0001.01.ENG Consulté le 6 décembre 2014

2. European Commission. Impact assessment report on the revision of the "Clinical Trials Directive" 2001/20/EC. 2012 http://ec.europa.eu/health/files/clinicaltrials/2012_07/ impact_assessment_part1_en.pdf Consulté le 6 décembre 2014 (72 pages)

3. Liddell K, Kompanje EJO, Lemaire F, et al. Recommendations in relation to the EU clinical trials directive and medical research involving incapacitated adults. Wien Klin Wochenschr 2006; 118: 183-91

4. Demarez JP. Le CPPRB, des origines à demain. La lettre du pharmacologue 2004; 18: 59-71

5. Lemaire F. La mission des CPP en France : ni éthique, ni scientifique ? Médecine Sci MS 2005; 21: 876-9

6. Wolzt M, Druml C, Leitner D, et al. Protocols in expedited review: tackling the workload of ethics committees. Intensive Care Med 2009; 35: 613-5

7. Loi n ${ }^{\circ}$ 2011-2012 du 29 décembre 2011 relative au renforcement de la sécurité sanitaire du médicament et des produits de santé. 2011-2012. 2011 http://www. legi france.gouv. fr Consulté le 6 décembre 2014

8. Décret n ${ }^{\circ}$ 2012-1244 du 8 novembre 2012 relatif au renforcement des dispositions en matière de sécurité des médicaments à usage humain soumis à autorisation de mise sur le marché et à la pharmacovigilance. 2012 http://www.legifrance.gouv.fr Consulté le 6 décembre 2014

9. Parlement Européen. Règlement (UE) No 1235/2010 du Parlement Européen et du Conseil du 15 décembre 2010 modifiant, en ce qui concerne la pharmacovigilance des médicaments à usage humain, le règlement $(\mathrm{CE})$ no 726/2004 établissant des procédures communautaires pour l'autorisation et la surveillance en ce qui concerne les médicaments à usage humain et à usage vétérinaire, et instituant une Agence européenne des médicaments, et le règlement (CE) no 1394/2007 concernant les médicaments de thérapie innovante. 2010

http://ec.europa.eu/health/files/eudralex/vol-1/reg_2010_ 1235/reg_2010_1235_fr.pdf Consulté le 6 décembre 2014 (16 pages)

10. Parlement Européen. Directive 2010/84/UE du Parlement Européen et du Conseil du 15 décembre 2010 modifiant, en ce qui concerne la pharmacovigilance, la directive 2001/83/CE instituant un code communautaire relatif aux médicaments à usage humain. 2010

http://ec.europa.eu/health/files/eudralex/vol-1/dir_2010_ 84/dir_2010_84_fr.pdf

Consulté le 6 décembre 2014 (26 pages)

11. Bras PL, Loth A. Rapport sur la gouvernance et l'utilisation des données de santé. 2013

http://www. social-sante.gouv.fr/IMG/pdf/Rapport_donnees_ de_sante_2013.pdf

Consulté le 6 décembre 2014 (128 pages)

12. Dahan M, Cahut Ch, Coste Ph. Evolution des CPP évaluant les projets de recherche impliquant la personne humaine, après la loi du 5 mars 2012. $2014 \mathrm{http://www.ladocumentationfrancaise.fr/var/}$ storage/rapports-publics/144000349/0000.pdf Consulté le 6 décembre 2014 (236 pages)

Correspondance et offprints : François Lemaire, 92 rue d'Alésia, 75014 Paris, France.

E-mail : f.lemaire@numericable.com 